\title{
Calculation of solid-state cargo fastener under the influence of longitudinal forces
}

\author{
Yadgor Ruzmetov ${ }^{1 *}$, Oksana Molchanova ${ }^{2}$, and Jamol Shihnazarov ${ }^{1}$ \\ ${ }^{1}$ Tashkent Railway Engineering Institute, Temirylchilar Str., 1, Tashkent, 100167, Uzbekistan \\ ${ }^{2}$ Ural State University of Railway Transport, Kolmogorov Str., 66, Yekaterinburg, 620034, Russia
}

\begin{abstract}
Cargo securing on a railway platform, elastic and retention fasteners, longitudinal transport force of inertia, forces in elastic fastenings. Purpose: The paper presents the calculation of the longitudinal transport force of inertia; determines the longitudinal force perceived by the elastic elements of the cargo securing; determines the reactions of retention bars depending on the number of bars and fasteners (nail); calculates the sum of all holding forces; calculates the forces in the elastic fasteners when exposed to longitudinal forces. Research methods: To determine the forces in the cargo securing devices, existing calculation methods are used. Moreover, the formulas of these techniques are presented in a form convenient for calculation. Main results: The paper proves that according to the existing method, the force in all elastic cargo fasteners under the influence of longitudinal forces has the same value, regardless of the different arrangement of the geometric parameters of the fasteners in space. The forces in them are more than 1.6 times higher than the permissible values. The calculations of forces in the elastic fasteners, which are performed by the specified method, are given. The results of the comparative analysis showed that the forces in all wire fasteners, in contrast to the existing calculation method, have different values. However, the strength of the second pair of wire fasteners of both one and the other direction is not ensured - the force in them more than two times exceeds the permissible value $(61 / 24.8 \approx 2.5)$, which will result in their destruction during the cargo transportation. Conclusions and their significance for the industry: Existing calculation methods do not provide guarantee strength of cargo fasteners. In the future, to calculate the fastening of cargo by shippers in the railway industry, a new calculation procedure should be applied for the allocation and fastening of cargo on the car.
\end{abstract}

\section{Introduction}

Cargo securing on rolling stock is a well-known problem [1 - 22]. So, for example, in [1], particular attention was paid to the calculation of the retention element (retention bar) of cargo securing. In [1], the recommended value of the force per one fastener (nail) is $\left[R_{\text {fas }}\right]=$ $1.5(\mathrm{kN})$. In $[1,2]$, it was established by experimental studies that the maximum amplitude

*Corresponding author: yadgor.ruzmetov@yandex.ru 
of oscillations of a car with a cargo reaches $25 \mathrm{~mm}$, and up to $35 \mathrm{~mm}$ in a vertical plane. During vibration with a frequency of $30 \mathrm{~Hz}$, the coefficient of friction between the contacting surfaces of the cargo and the floor of the car decreases by almost $30 \%$, which is the reason for the longitudinal and transverse shear of cargo in the car. However, these results were not taken into account in [11]. In [5], using the concepts of "shear" and "holding" forces for the first time, an example is given of calculating the fastening of piece cargo on a platform when the shear of a cargo is held by a wooden bar nailed to the floor of the car. The results of studies [5] also did not find application in [11]. The incorrectness of the formulas in [11], according to which the calculation of flexible cargo securing elements is carried out, are described in [12 - 20, 22]. In [12-20], it was noted that the determination of the forces in the elastic elements of cargo fastenings by the method of [11] was reduced to solving the problem of a static definable system, which does not fully comply with the basic principles of solving problems of a static indefinable system in mechanics [14]. Therefore, the formulas for calculating the forces in wire cargo fasteners given in [11] are true only for one pair of fasteners with the same topology and nothing more. In fact, wire cargo fasteners have various topologies, geometric parameters and preliminary twist forces. The determination of the force in wire fasteners becomes the static indefinable task of the mechanical system "cargo-fastener-car" [14]. Therefore, the urgency of the technical problem of calculating the fastening of cargo transported on a railway platform becomes obvious. The following example will clearly demonstrate the drawbacks of the existing methodology for calculating the forces in the wire fastenings of cargo [11] and, in our opinion, is of practical interest to transport science.

\section{Object}

- calculate the longitudinal transport force of inertia and calculate the friction force of adhesion from the gravity of a cargo;

- calculate the longitudinal force perceived by the elastic fastening elements of a cargo and find the friction force of adhesion from the forces of the preliminary twists of the fastening wire;

- determine the reactions of the retention bars depending on the number of bars and fasteners (nail) and calculate the holding forces that prevent the shear of the cargo along the car;

- calculate the sum of all the holding forces that are perceived by the elastic fastening elements and retention bars; determine the difference between the shear and holding forces as a longitudinal force;

- calculate the forces in the elastic fasteners when exposed to longitudinal forces.

\section{Method of research}

The study relies on the results of calculations of longitudinal forces obtained by the authors of the paper and on the determination of forces in cargo fasteners, using the formula of the existing calculation method [11]. Moreover, the formulas of these methods are presented by the authors of the paper in a form convenient for calculation. 


\section{Research results}

\subsection{Mathematical representation of forces}

Below there are the results of calculations of forces in flexible elements of cargo securing on a railway platform (Fig. 1).

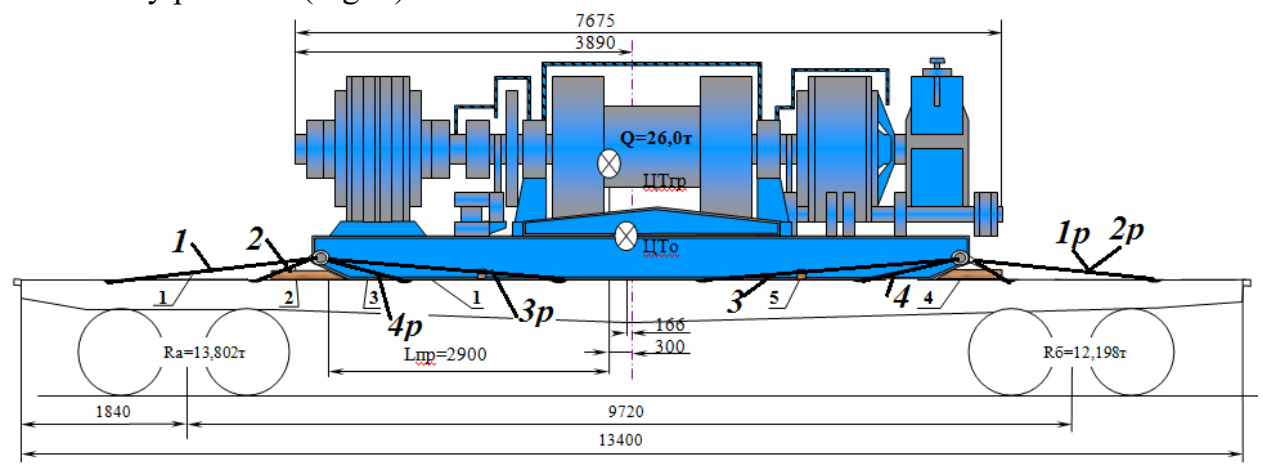

a)

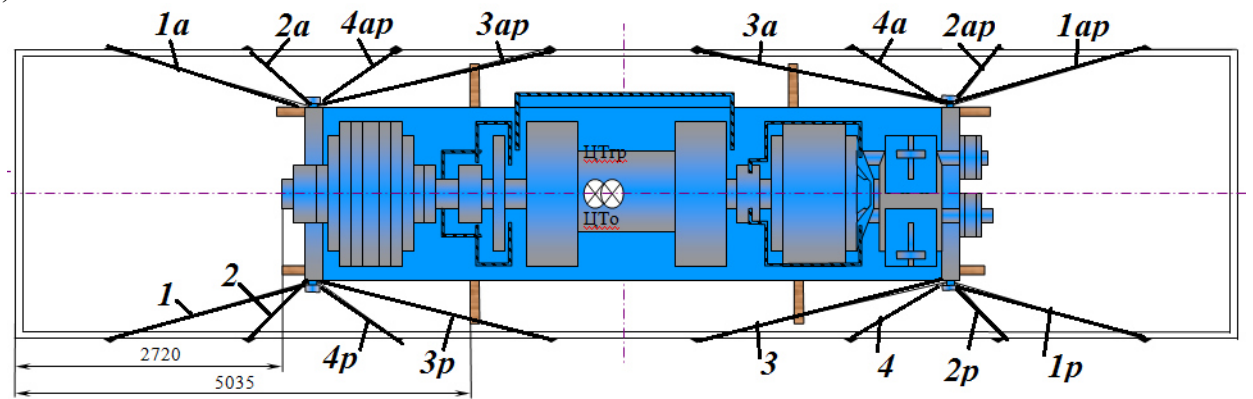

b)

Fig. 1. Scheme of allocation and fastening of cargo on the car ((a) - main view; (b) - top view)

In fig. 1, 1 - 4, 1a-4a;1p - 4p, 1ap - 4ap - numbers of elastic fasteners of the one and the other direction.

The calculation of the longitudinal forces perceived by elastic and retention fasteners according to the formulas given in [6,12-20] and using the Mathcad system [23] will be described in the following sequence.

Longitudinal transport force of inertia $I_{e x}$ (see formula (1.19) in [14]):

$$
I_{e x}=k_{\text {tol. } x} G \text {, }
$$

where $k_{\text {tol } x}=a_{e x} / \mathrm{g}$ - the coefficient of longitudinal dynamics of the car and/or longitudinal translational acceleration of a car with a cargo in fractions of $g$ (usually taken within $1.2 \div 2$ ) [14];

$a_{e x}$ longitudinal translational acceleration of a car $\left(\mathrm{m} / \mathrm{s}^{2}\right)$ (usually take $1.6 \mathrm{~g}$ );

$G$ - cargo gravity (for the considered example - $260(\mathrm{kN})$ ).

Substituting the initial data, we obtain $(\mathrm{kN})$ :

$I_{e x}=k_{\text {tol. } x} G=1.6 \cdot 260=416$.

Friction force during coupling [14]:

$$
F_{\text {fr.coup. } x}=f_{\text {coup. }} G \text {, }
$$

where $f_{\text {coup }}$ - coefficient of friction during coupling (usually "metal - wood" 0.45 ). 
Friction force during coupling is $(\mathrm{kN})$ :

$F_{\text {fr.coup } x}=f_{\text {coup. }} G=0.45 \cdot 260=117$.

Longitudinal shear force (see formula (3.9) in [14]) $(\mathrm{kN})$ :

$F_{\text {shear } x}=F_{x}=I_{e x}=416$.

Determination of the longitudinal force perceived by the elastic fastening elements of the cargo, taking into account only the friction forces of coupling, is performed according to the formula (4.6) in [5]:

$$
\Delta F_{x}=F_{\text {shear } x}-F_{\text {fr.coup } x} .
$$

Taking into account the initial data obtained in (1) and (2), we have $(\mathrm{kN})$ :

$\Delta F_{x}=F_{\text {shear } x}-F_{\text {fr.coup } x}=416-117=299$.

\subsection{Results of calculations of forces in fasteners}

Calculation of the force in the elastic fastening elements according to the recommended formulas is feasible in the following sequence.

The projections of the elastic fastening forces on the longitudinal axis, taking into account the forces of the preliminary twists of the fastening wire $R 0$, are found by the formula [14]:

$$
R 0_{k x}=R 0 \times\left(\frac{a_{i}}{l_{i}}+\frac{a_{i a}}{l_{i a}}\right) ; \quad R 0_{k x}=R 0 \times\left(\frac{a_{i p}}{l_{i p}}+\frac{a_{i a p}}{l_{i a p}}\right) \quad(k=1.2 ; \quad i=\overline{1.6}) .
$$

Here $R 0=20$ is the accepted average value of the tension (force) of the preliminary twists of the fastening wire $(\mathrm{kN}) ; a_{i}$ and $l_{i}, a_{i a}$ and $l_{i a} ; a_{i p}$ and $l_{i p}, a_{i a p}$ and $l_{i a p}$ - values (calculated according to the drawing) of the projection of the elastic fasteners, both one and the other direction, on the longitudinal axis of the car and the length of these fasteners (m).

For this example, these geometric parameters of the securing devices are summarized in Table 1.

\begin{tabular}{|c|c|c|c|c|c|}
\hline $\begin{array}{l}\text { Designation } \\
\text { of the }\end{array}$ & Direction of & \multicolumn{4}{|c|}{$\begin{array}{l}\text { The values of the projections of the securing devices } \\
\text { and their length }(\mathrm{m})\end{array}$} \\
\hline$a_{i}$ & \multirow{4}{*}{ One direction } & 1.955 & 0.557 & 2.043 & 0.819 \\
\hline$l_{i}$ & & 2.059 & 0.854 & 2.143 & 1.044 \\
\hline$a_{i a}$ & & 1.955 & 0.557 & 2.043 & 0.819 \\
\hline$l_{i a}$ & & 2.059 & 0.854 & 2.143 & 1.044 \\
\hline$a_{i p}$ & \multirow{4}{*}{$\begin{array}{l}\text { The other } \\
\text { direction }\end{array}$} & 1.955 & 0.469 & 2.217 & 0.732 \\
\hline$l_{i p}$ & & 2.059 & 0.799 & 2.31 & 0.977 \\
\hline$a_{\text {iap }}$ & & 1.868 & 0.469 & 2.217 & 0.732 \\
\hline$l_{\text {iap }}$ & & 1.977 & 0.799 & 2.31 & 0.977 \\
\hline
\end{tabular}

Table 1. Geometry of the securing devices $(\mathrm{m})$

Substituting the initial data from Table 1 in the last formula and taking into account the symmetrical placement of the cargo relative to the longitudinal axis of the car, both one and the other direction, we obtain $(\mathrm{kN})$ :

$$
\begin{aligned}
& R 0_{1 x}=20 \times 2 \times\left(\frac{1.995}{2.059}+\frac{0.557}{0.854}+\frac{0.52}{1.06}+\frac{2.043}{2.143}+\frac{0.819}{1.044}\right)= \\
& =40 \times(0.949+0.652+0.953+0.785)=40 \times 3.339=133.591 \\
& R 0_{2 x}=20 \times\left(\frac{1.995}{2.059}+\frac{0.469}{0.799}+\frac{2.217}{2.31}+\frac{0.732}{0.977}+\frac{1.868}{1.977}+\frac{0.469}{0.799}+\frac{2.217}{2.31}+\frac{0.732}{0.977}\right)= \\
& =20 \times(0.949+0.587+0.96+0.749+0.945+0.587+0.96+0.749)=20 \times 6.487=129.741 .
\end{aligned}
$$

Calculation of the difference in the projection of the force of the preliminary twists of 
the fastening wire on the longitudinal axis, both one and the other direction $(\mathrm{kN})$ :

$$
F R 0_{x 0}=R 0_{1 x}-R 0_{2 x}=133.591-129.741=3.849 \text {. }
$$

Determination of the projection of the force (elastic forces) of the preliminary twists of the fastening wire $R 0$ on the vertical axis of one and the other direction (see formula (19) in [20]).

$$
R 0_{k z}=R 0 \times\left(\frac{h_{i}}{l_{i}}+\frac{h_{i a}}{l_{i a}}\right) ; \quad R 0_{k z}=R 0 \times\left(\frac{h_{i p}}{l_{i p}}+\frac{h_{i a p}}{l_{i a p}}\right) \quad(k=\overline{1.2} ; \quad i=\overline{1.6}),
$$

where $h_{i}$ and $l_{i}, h_{i a}$ and $l_{i a}, h_{i p}$ and $l_{i p}, h_{i a p}$ and $l_{i a p}$ - values (calculated according to the drawing) of the projection of the elastic fasteners, both one and the other direction, on the vertical axis of the car and the length of these fasteners $(\mathrm{m})$.

For this example, these geometrical parameters of the securing devices are summarized in Table 2.

\begin{tabular}{|c|c|c|c|c|c|}
\hline $\begin{array}{l}\text { Designation of } \\
\text { the securing }\end{array}$ & $\begin{array}{l}\text { Direction } \\
\text { of }\end{array}$ & \multicolumn{4}{|c|}{$\begin{array}{l}\text { The values of the projections of the securing devices and } \\
\text { their length }(\mathrm{m})\end{array}$} \\
\hline$h_{i}$ & \multirow{4}{*}{$\begin{array}{c}\text { One } \\
\text { direction }\end{array}$} & 0.302 & 0.302 & 0.302 & 0.302 \\
\hline$l_{i}$ & & 2.059 & 0.854 & 2.143 & 1.044 \\
\hline$h_{i a}$ & & 0.302 & 0.302 & 0.302 & 0.302 \\
\hline$l_{i a}$ & & 2.059 & 0.854 & 2.143 & 1.044 \\
\hline$h_{i p}$ & \multirow{4}{*}{$\begin{array}{l}\text { The other } \\
\text { direction }\end{array}$} & 0.302 & 0.302 & 0.302 & 0.302 \\
\hline$l_{i p}$ & & 2.059 & 0.799 & 2.31 & 0.977 \\
\hline$h_{i a p}$ & & 0.302 & 0.302 & 0.302 & 0.302 \\
\hline$l_{\text {iap }}$ & & 1.977 & 0.799 & 2.31 & 0.977 \\
\hline
\end{tabular}

Table 2. Geometry of the securing devices (m)

Substituting the initial data from Table 2 in the last formula and taking into account the symmetrical placement of the cargo relative to the longitudinal axis of the car, of one or the other direction, we obtain the values of the projection of elastic forces from preliminary twists of the fastening wire on the vertical axis $(\mathrm{kN})$ :

$$
\begin{aligned}
& R 0_{1 z}=20 \times 2 \times\left(\frac{0.302}{2.059}+\frac{0.302}{0.854}+\frac{0.302}{2.143}+\frac{0.302}{1.044}\right)= \\
& =40 \times(0.412+0.278+0.858+0.633)=40 \times 0.932=37.28 \\
& R 0_{2 z}=20 \times\left(\frac{0.302}{2.059}+\frac{0.302}{0.799}+\frac{0.302}{2.31}+\frac{0.302}{0.977}+\frac{0.302}{1.977}+\frac{0.302}{0.799}+\frac{0.302}{2.31}+\frac{0.302}{0.977}\right)= \\
& =20 \times(0.147+0.378+0.131+0.31+0.153+0.378+0.131+0.31)=20 \times 1.937=38.753 .
\end{aligned}
$$

Determination of the friction force of coupling from the force of preliminary twists of the fastening wire $R 0$ on the vertical axis (see formula (11a) in [20]):

$$
F R 0_{\text {coup. } k x}=f_{\text {coup. }} \times \sum_{i=1}^{n_{\mathrm{p}}} R 0_{i z} \quad(k=\overline{1.2} ; \quad i=\overline{1.6}) .
$$

Calculation of the friction force of coupling from preliminary twists of the fastening wire, both one and the other direction $(\mathrm{kN})$ :

$$
\begin{aligned}
& F R 0_{\text {coup. } 1 x}=f_{\text {coup. }} \times R 0_{z 1 x}=0.45 \times 37.28=14.912 \\
& F R 0_{\text {coup. } 2 x}=f_{\text {coup. }} \times R 0_{z 2 x}=0.45 \times 38.753=15.501 .
\end{aligned}
$$

Calculation of the sum of the friction force of coupling from preliminary twists of the fastening wire, both one and the other direction $(\mathrm{kN})$ :

$$
\Delta F R 0_{\text {coup. } 1 x}=F R 0_{\text {coup } .1 x}+F R 0_{\text {coup. } 2 x}=14.912+15.501=30.413 .
$$

Thus, the calculation results, as in [14], proved that the projections of the cargo securing devices on the vertical axis affect the magnitude of the friction force along the longitudinal 
axis of the car. In this case, the value of the holding forces increases.

We will determine the holding force along the car from the forces of the preliminary twists of the fastening wire, both one and the other direction, according to our formula:

$$
F R 0_{\text {coup.ret } x 0}=F_{\text {fr.coup. } x}+F R 0_{x 0}+F R 0_{\text {fr.coup } x 0} \text {. }
$$

Substituting the initial data calculated by formulas (1), (4) and (6) in (7), we obtain $(\mathrm{kN})$ :

$$
F R 0_{\text {coup.ret. } x 0}=F_{\text {fr.coup } x}+F R 0_{x 0}+F R 0_{\text {fr.coup } x 0}=104+3.849+30.413=138.262 .
$$

Finding the reaction of retention bars according to the formulas (see formula (46) Appendix 3 to SMGS [11]):

$$
R_{\text {bar. } x}=k_{1} n_{\text {nail. } x} n_{\text {bar. } x}\left[R_{\text {nail }}\right] \text {, }
$$

where $k_{1}=0.6-$ the coefficient of strength of fastening retention bars, taking into account the condition of the floor of the car, pes. (usually take $0.5-0.6$ ); ${ }^{n}$ nail.x $=12$ and $n_{\text {bar. } x}=2$ - the number of fasteners (nails) and retention bars taken in accordance with the scheme of allocation and fastening of cargo on the car, pcs. (see Fig. 1); $\left[R_{\text {nail }}\right]=1.08-$ the permissible value of force per one fastener (nail) (kN) (tab. 32 Appendix 3 to SMGS [11]), and in [1], it is recommended to take $\left[R_{\text {nail }}\right]=1.5$.

Substituting the received data in (10), we obtain the reaction of the retention bars with the taken number of nails $(\mathrm{kN})$ :

$$
R_{\text {bar. } x}=k_{1} n_{\text {nail. } x} n_{\text {bar. } x}\left[R_{\text {nail }}\right]=0.6 \cdot 12 \cdot 2 \cdot 1.08=15.552 \text {. }
$$

Determination of the sum of all the "holding" forces along the car, taking into account the reaction of the retention bars, both one and the other direction:

$$
F R 0_{\text {fr.coup } x}=F R 0_{\text {fr.coup } x}+F_{\text {bar } x} \text {. }
$$

Substituting the initial data calculated by formulas (7) and (8) in (9), we obtain $(\mathrm{kN})$ :

$$
F R 0_{\text {fr.coup. } x}=F R 0_{\text {fr.coup. } x}+F_{\text {bar. } x}=104+15.552=119.552 \text {. }
$$

The longitudinal force perceived by the cargo securing devices and/or the difference between the shear and holding forces [15]:

$$
\Delta F R 0_{\text {bar.coup } x}=F_{\text {shear } x}-F R 0_{\text {fr.coup } x} \text {. }
$$

Substituting the initial data calculated by formulas (1) and (9) in (10), we obtain $(\mathrm{kN})$ :

$$
\Delta F R 0_{\text {bar.coup. } x}=F_{\text {shear. } x}-F R 0_{\text {fr.coup. } x}=416-119.552=296.448 \text {. }
$$

A positive sign of longitudinal force means that the shear force is larger in value than the holding force. In this case, the cargo will obviously shift along the car, which is accompanied by the pulling out of fasteners (nail) of the retention bars and the turning of these bars relative to the face farthest from the end surface of the cargo.

\subsection{Calculation of a force in flexible cargo securing devices according to the existing method [11]}

The forces in the elastic fasteners of the cargo of one or the other direction under the action of longitudinal forces are determined by the formulas (see formula (39) in [11]), which, for the convenience of calculation, are presented in the form:

$$
R_{k x}=\frac{\Delta F_{\text {bar.coup } x}}{a} \quad(k=\overline{1.2} ; \quad a \in\{b 1, b 2\}),
$$


where $\Delta F_{\text {bar.coup.x }}$ - longitudinal force perceived by elastic and retention fasteners (see formula (10), where $\Delta F_{\text {bar.coup. } x}=296.448(\mathrm{kN})$ );

$b_{1}$ and $b_{2}$ - dimensionless constant numbers, which are determined through the specified values of the physico-geometric parameters of wire fasteners in the form:

$$
\begin{aligned}
& b 1=\left(f_{\text {coup. }} \times \frac{h_{i}}{l_{i}}+\frac{a_{i}}{l_{i}}\right)+\left(f_{\text {coup. }} \times \frac{h_{i p}}{l_{\text {ip }}}+\frac{a_{i p}}{l_{\text {ip }}}\right) ; \\
& b 2=\left(f_{\text {coup. }} \times \frac{h_{\text {ia }}}{l_{\text {ia }}}+\frac{a_{\text {ia }}}{l_{\text {ia }}}\right)+\left(f_{\text {coup. }} \times \frac{h_{\text {iap }}}{l_{\text {iap }}}+\frac{a_{\text {iap }}}{l_{\text {iap }}}\right) .
\end{aligned}
$$

Here $h_{i}, a_{i}$ and $l_{i} ; h_{i p}, a_{i p}$ and $l_{i p} ; h_{i a}, a_{i a}$ and $l_{i a} ; h_{i a p}, a_{i a p}$ and $l_{i a p}$ - the values (calculated according to the drawing) of the projection of the elastic fasteners of one or the other direction on the vertical and longitudinal axis of the car, respectively, and the length of these fasteners $(\mathrm{m})$.

For the example under study, these geometric parameters of the securing devices are given in Table 1 and 2.

Substituting the data from tables 1 and 2 in formulas (12) and (13), taking into account that the coefficient of friction of coupling $f_{\text {coup }}=0.45$, we obtain:

$$
\begin{aligned}
& b 1=\left(\begin{array}{l}
\left(0.45 \times \frac{0.302}{2.059}+\frac{1.995}{2.059}\right)+\left(0.45 \times \frac{0.302}{0.854}+\frac{0.557}{0.854}\right)+\left(0.45 \times \frac{0.302}{2.14}+\frac{2.043}{2.143}\right)+ \\
+\left(0.45 \cdot \frac{0.302}{1.044}+\frac{0.819}{1.044}\right)+\left(0.45 \times \frac{0.302}{2.059}+\frac{1.995}{2.059}\right)+\left(0.45 \times \frac{0.302}{0.799}+\frac{0.469}{0.799}\right)+ \\
+\left(0.45 \times \frac{0.302}{2.31}+\frac{2.217}{2.31}\right)+\left(0.45 \cdot \frac{0.302}{0.977}+\frac{0.732}{0.977}\right)
\end{array}\right)=7.425 \\
& b 2=\left(\begin{array}{l}
\left(0.45 \times \frac{0.302}{2.059}+\frac{1.995}{2.059}\right)+\left(0.45 \times \frac{0.302}{0.854}+\frac{0.557}{0.854}\right)+\left(0.45 \times \frac{0.302}{2.14}+\frac{2.043}{2.143}\right)+ \\
+\left(0.45 \cdot \frac{0.302}{1.044}+\frac{0.819}{1.044}\right)+\left(0.45 \times \frac{0.302}{1.977}+\frac{1.868}{1.977}\right)+\left(0.45 \times \frac{0.302}{0.799}+\frac{0.469}{0.799}\right)+ \\
+\left(0.45 \times \frac{0.302}{2.31}+\frac{2.217}{2.31}\right)+\left(0.45 \cdot \frac{0.302}{0.977}+\frac{0.732}{0.977}\right)
\end{array}\right)=6.891 .
\end{aligned}
$$

Given the obtained values of dimensionless constant numbers $b_{1}, b_{2}$ and the value of the longitudinal forces $\Delta F_{\text {bar.coup. } x}=296.448(\mathrm{kN})$, and also taking into account the asymmetry of the location of the securing devices relative to the longitudinal axis of one or the other direction, we obtain by formula $(11)(\mathrm{kN})$ :

$$
R_{1 x}=\frac{\Delta F_{\text {bar.coup } . x}}{b 1}=\frac{296.448}{7.425}=39.925 ; \quad R_{2 x}=\frac{\Delta F_{\text {bar.coup } . x}}{b 2}=\frac{296.448}{6.891}=40.821 .
$$

Thus, it becomes obvious that the force in all the elastic fasteners of the cargo of one or the other direction under the influence of longitudinal forces has the same value, regardless of the different arrangement of the geometric parameters of the fasteners in space. This is not true at all, since different lengths of elastic fasteners must correspond to different values of force in these fasteners. In addition, the sum of the forces in all the elastic fasteners is much larger (for example, 1.6 times $(40 / 24.8 \approx 1.6))$ than the allowable value $(24.8(\mathrm{kN})$ ). Therefore, we can conclude that according to the existing calculation method, the strength of fasteners with a diameter of $d=6(\mathrm{~mm})$ and the number of threads $n=8(\mathrm{pcs})$ is not provided. As can be seen, the calculation results proved that the method of calculating the forces in the cargo securing devices [11] does not ensure the safety of the transportation 
process.

\subsection{Determination of the cargo securing force according to the formulas from Appendix 3 [11]}

The forces in the elastic securing devices (bracing wires) when exposed to longitudinal forces are determined by the formula (1) from Appendix 3 in [11]:

$$
R_{i x}=Z_{k x} \times \frac{n_{i}}{l_{i}} \times \frac{a_{i}}{l_{i}} ; R_{i p x}=Z_{k x} \times \frac{n_{i p}}{l_{i p}} \times \frac{a_{i p}}{l_{i p}} \quad(k=\overline{1.2} ; \quad i=\overline{1.6}),
$$

where $Z_{k x}$ - the physical parameter determined by the formula (2) from Appendix 3 in [12], which we presented in the following form:

$$
Z_{1 x}=\frac{\Delta F_{\text {bar.coup } . x}}{C 0_{\text {coup. } .1 x}} \text { and } Z_{2 x}=\frac{\Delta F_{\text {bar.coup } . x}}{C 0_{\text {coup } .2 x}} .
$$

Where $\Delta F_{\text {bar.coup.x }}$ - longitudinal force acting on elastic and retention fasteners (see formula (10)); $f_{\text {coup }} \quad-$ friction coefficient of coupling (usually 0.45 ); $c 0_{\text {coup.1x }}$ and $c 0_{\text {coup. } 2 x}$ - additional parameters of elastic fasteners, which are determined by the formula (2) from Appendix 3 in [11].

We presented additional parameters of the elastic fasteners $c 0_{\text {coup. } x}$ along the car with the friction coefficient of coupling according to the formulas obtained from (2) from Appendix 3 in [11] in the following form:

$$
\begin{aligned}
& c 0_{\text {coup. } 1 x}=\sum_{i=1}^{n_{\mathrm{p}}} \frac{n_{i}}{l_{i}} \times\left(f_{\text {coup. }} \times \frac{h_{i}}{l_{i}}+\frac{a_{i}}{l_{i}}\right) \times \frac{a_{i}}{l_{i}}+\sum_{i=1}^{n_{\mathrm{p}}} \frac{n_{i a}}{l_{\text {ia }}} \times\left(f_{\text {ccoup. }} \times \frac{h_{i a}}{l_{\text {ia }}}+\frac{a_{i a}}{l_{\text {ia }}}\right) \times \frac{a_{i a}}{l_{\text {ia }}} ; \\
& c 0_{\text {coup } .2 x}=\sum_{i=1}^{n_{\mathrm{p}}} \frac{n_{i}}{l_{i}} \times\left(f_{\text {coup. }} \times \frac{h_{i p}}{l_{i p}}+\frac{a_{i p}}{l_{i p}}\right) \times \frac{a_{i p}}{l_{i p}}+\sum_{i=1}^{n_{\mathrm{p}}} \frac{n_{i p}}{l_{i p}} \times\left(f_{\text {coup. }} \times \frac{h_{\text {iap }}}{l_{\text {iap }}}+\frac{a_{\text {iap }}}{l_{\text {iap }}}\right) \times \frac{a_{\text {iap }}}{l_{\text {iap }}} .
\end{aligned}
$$

Note that here the geometric parameters of the fasteners have the same values as shown in Table 1 and 2.

Substituting the initial data from the tables 1 and 2 in the last formulas with $f_{\text {coup. }}=$ $0.45, d_{i}=6(\mathrm{~mm}), n_{i}=8(\mathrm{pcs})$, we obtain $(1 / \mathrm{m})$ :

$$
c 0_{\text {coup } .1 x}=\left(\begin{array}{l}
\frac{8}{2.059}\left(0.45 \times \frac{0.302}{2.059}+\frac{1.995}{2.059}\right) \frac{1.995}{2.059}+\frac{8}{0.854}\left(0.45 \times \frac{0.302}{0.854}+\frac{0.557}{0.854}\right) \frac{0.557}{0.854}+ \\
+\frac{8}{2.143}\left(0.45 \times \frac{0.302}{2.14}+\frac{2.043}{2.143}\right) \frac{2.043}{2.143}+\frac{8}{1.044}\left(0.45 \cdot \frac{0.302}{1.044}+\frac{0.819}{1.044}\right) \frac{0.819}{1.044}+ \\
+\frac{8}{2.059}\left(0.45 \times \frac{0.302}{2.059}+\frac{1.995}{2.059}\right) \frac{1.995}{2.059}+\frac{8}{0.799}\left(0.45 \times \frac{0.302}{0.799}+\frac{0.469}{0.799}\right) \frac{0.469}{0.799}+ \\
+\frac{8}{2.31}\left(0.45 \times \frac{0.302}{2.31}+\frac{2.217}{2.31}\right) \frac{2.217}{2.31}+\frac{8}{0.977}\left(0.45 \cdot \frac{0.302}{0.977}+\frac{0.732}{0.977}\right) \frac{0.732}{0.977}
\end{array}\right)=35.169 ;
$$




$$
c 0_{\text {coup } .2 x}=\left(\begin{array}{l}
\frac{8}{2.059}\left(0.45 \times \frac{0.302}{2.059}+\frac{1.995}{2.059}\right) \frac{1.995}{2.059}+\frac{8}{0.854}\left(0.45 \times \frac{0.302}{0.854}+\frac{0.557}{0.854}\right) \frac{0.557}{0.854}+ \\
+\frac{8}{2.143}\left(0.45 \times \frac{0.302}{2.14}+\frac{2.043}{2.143}\right) \frac{2.043}{2.143}+\frac{8}{1.044}\left(0.45 \cdot \frac{0.302}{1.044}+\frac{0.819}{1.044}\right) \frac{0.819}{1.044}+ \\
+\frac{8}{1.977}\left(0.45 \times \frac{0.302}{1.977}+\frac{1.868}{1.977}\right) \frac{1.868}{1.977}+\frac{8}{0.799}\left(0.45 \times \frac{0.302}{0.799}+\frac{0.469}{0.799}\right) \frac{0.469}{0.799}+ \\
+\frac{8}{2.31}\left(0.45 \times \frac{0.302}{2.31}+\frac{2.217}{2.31}\right) \frac{2.217}{2.31}+\frac{8}{0.977}\left(0.45 \cdot \frac{0.302}{0.977}+\frac{0.732}{0.977}\right) \frac{0.732}{0.977}
\end{array}\right)=33.695 .
$$

Substituting the obtained values of $c 0_{\text {coup. } x}$ in the formula (15), we obtain $(\mathrm{kN} \cdot \mathrm{m})$ :

$Z_{1 x}=\frac{\Delta F_{\text {bar.coup } . x}}{C 0_{\text {coup. } 1 x}}=\frac{296.448}{35.169}=8.429 ; Z_{2 x}=\frac{\Delta F_{\text {bar.coup } . x}}{C 0_{\text {coup. } 2 x}}=\frac{296.448}{33.695}=8.798$.

The results of the calculation of the forces in the cargo securing elements according to the formula (14), taking into account the calculated data obtained by the formulas (10), (14) - (17), are summarized in Table $3(\mathrm{kN})$.

Table 3. Calculated forces of the securing elements in either direction $(\mathrm{kN})$

\begin{tabular}{|c|c|c|c|c|c|}
\hline $\begin{array}{c}\text { Designations } \\
\text { of forces in } \\
\text { the securing } \\
\text { elements }\end{array}$ & $\begin{array}{c}\text { Direction } \\
\text { of } \\
\text { securing }\end{array}$ & \multicolumn{4}{|c|}{ Forces in the securing elements $(\mathrm{kN})$} \\
\hline \multirow{2}{*}{$\frac{R_{i x}}{R_{\text {iax }}}$} & $\begin{array}{c}\text { One } \\
\text { direction }\end{array}$ & $\frac{R_{1 x}}{R_{1 a x}}$ & $\frac{R_{2 x}}{R_{2 a x}}$ & $\frac{R_{3 x}}{R_{3 a x}}$ & $\frac{R_{4 x}}{R_{4 a x}}$ \\
\cline { 3 - 6 } & $\frac{31.087}{31.087}$ & $\frac{51.538}{51.538}$ & $\frac{30}{30}$ & $\frac{50.7}{50.7}$ \\
\hline$R_{i p x}$ & \multirow{2}{*}{$\begin{array}{c}\text { The other } \\
\text { direction }\end{array}$} & $\frac{R_{p 1 x}}{R_{\text {iapx }}}$ & $\frac{R_{2 p x x}}{R_{2 a p x}}$ & $\frac{R_{3 p x}}{R_{3 a p x}}$ & $\frac{R_{4 p x}}{R_{4 a p x}}$ \\
\cline { 3 - 6 } & & $\frac{32.446}{33.647}$ & $\frac{57.715}{57.715}$ & $\frac{29.252}{29.252}$ & $\frac{53.99}{53.99}$ \\
\hline
\end{tabular}

An analysis of the results of the calculated values of the forces in the elastic fasteners by the formulas (1) and (2) from Appendix 3 in [11] makes it possible to note that the forces in all wire fasteners (bracing wires), in contrast to the existing calculation method (see formula (11)), have different values, which correspond to reality. In addition, the forces in all wire fasteners with a diameter of $d=6 \mathrm{~mm}$ and the number of threads $n=8$ (pcs), except for the second pair of wire fasteners of both one and the other direction, under the action of longitudinal forces were less than permissible value $(24.8(\mathrm{kN}))$. However, the strength of the second pair of wire fasteners of both one and the other direction is not ensured - they will be destroyed during the transportation of cargo.

\section{Conclusions}

Summarizing the research results, we note the following. The sequence of calculating the longitudinal forces perceived by the wire fasteners of the cargo is described, which made it possible to find the forces in the wire fasteners of the cargo according to the existing methods for calculating the fastening $[5,11,14]$.

It is specifically noted that according to the existing method (see formula (11)), the 
force in all elastic cargo fasteners of one or the other direction under the influence of longitudinal forces has the same value, regardless of the different arrangement of the geometric parameters of the fasteners in space. This is not true, since different values of the force in these fasteners must correspond to different lengths of elastic securing elements, thereby confirming the senselessness of using the existing method for calculating cargo securing [11] in order to ensure guarantee safety of the transportation process.

It was established that the calculated values of the forces in the elastic fasteners according to formulas (1) and (2) from Appendix 3 in [11] made it possible to note that the forces in all wire fasteners, in contrast to the existing calculation method (see formula (11)), have different values, which correspond to reality. However, this method also has drawbacks: the strength of the second pair of wire fasteners of both one and the other direction is not ensured - the force in them is more than two times higher than the allowable one $(61 / 24.8 \approx 2.5)$, which will result in their destruction during cargo transportation.

Given the drawbacks of the existing methods for calculating the forces in the elastic securing elements, in the future, a new calculation method for the placement and fastening of cargo on the car should be applied for the calculation of cargo securing [14].

\section{References}

1. N. Anderson, P. Andersonet al., Equipment for Rational Securing of Cargo on railway Wagons / VINNOVA Report/Rapport VR 2004:05 (2004) http://www.vinnova.se/upload / EPiStorePDF /vr-04-05.pdf.

2. M. Johanson, P. Andersson. Equipment for Efficient Cargo Securing and Ferry Fastening of Vehicles/NVF-rapporter/V ä gverket, http://www.nvfnorden.org/lisalib/getfile.aspx?itemid=1593

3. Driver's Handbook Cargo Securement. A Guide to the North American Cargo Securement Standard. $\quad$ http://www.highwaystarmagazine.com/images /Driver_Handbook.pdf

4. European Best Practice Guidelines on Cargo Securing for Road Transport. 208 p. http://ec.europa.eu/transport/roadsafety/vehicles/best_practice_guidelines_en.htm

5. K.L. Komarov, A.F. Yashin. Teoreticheskaia makhanika $v$ zadachakh gruzovykh perevozok. (Novosibirsk, Nauka Publ., 2004)

6. J.D. Priddy, R.A. Jones, Experimental Determination of the Effect of Cargo Variations on Roll Stability, SAE Technical Paper No. 2005-01-3516, (2005).

7. J.R. Billing Industry Practices for Loading and Securing Cargo in Vans and other Closed Trucks, (Ontario Trucking Association, 2006).

8. E. Hildebrand, M. Balsom, F.R. Wilson, Impact of Wind Forces on Heavy Truck Stability, Transportation Research Board Annual Meeting 2006, Paper \#06-1232. (2006)

9. Load Securement for Palletized Freight in Closed Trucks - From an Enforcement Perspective. dPE, Inc. "The Science of Preventing Freight Damage". January 2007. 50 p.

10. Vertical cylinder handling and transportation, AIGA 038/06. 24 p., http://www.asiaiga.org.

11. Technicheskie usloviia razmeshcheniia i krepleniia gruzov. Prilozdeniie 3. Retrieved February 5, 2020, from http://osjd.org/doco/public/ru

12. Kh. Turanov, E. Timukhina. Transport Problems, 3(3), 69-76 (2008).

13. Kh. Turanov. Transport Problems, 4(1), 77-86 (2009). 
14. Kh. Turanov. Teoreticheskaia makhanika $\mathrm{v}$ zadachakh gruzovykh perevozok. Monographiia (Novosibirsk: Nauka Publ. 2009)

15. Kh. Turanov, V. Olentsevich, Transport Problems, 5(1), 23-32 (2010).

16. Kh. Turanov, O. Portnova, (2010). Transport Problems, 5(2), 71-82 (2010)

17. Kh. Turanov, E. Timukhina. Transport Problems, 6(2), 79-88 (2011).

18. Kh. Turanov, E. Timukhina. Transport Problems, 6(3), 27-39. (2011)

19. Turanov, Kh.: Analytical investigation of cargo motion lengthwise the wagon under the action of plane force system. Global J. Res. Eng.: Mech. Mech. Eng. 13(X), 7-16 (2013). Version 1.0, New York

20. Kh. Turanov, Science and Technology. 5(1), 5-14 (2015). doi: 10.5923/j.scit.2015 0501.02

21. Kh. Turanov, Y. Ruzmetov, A.V. Yakushev Proceedings of Petersburg Transport University, 16 (3), 455-468 (2019). DOI: 10.20295/1815-588X-2019-1-455-468.

22. Kh. Turanov, Y. Ruzmetov, N. Vlasova E3S Web of Conferences, 135 (2019). https://doi.org/10.1051/e3sconf/201913502006

23. D.V. Kiryanov. Samouchitel Mathcad 13 [Self-instruction Mathcad 13]. (St. Petersburg, BKhV-Peterburg Publ. 2006) 